\title{
Discovery of the New Element Z=117 and Confirmation of 115
}

\author{
Yu. Ts. Oganessian ${ }^{1}$, J. H. Hamilton ${ }^{2}$ and V. K. Utyonkov ${ }^{1}$ for the collaboration \\ ${ }^{1}$ Joint Institute for Nuclear Research, RU-141980, Dubna, Russian Federation, \\ ${ }^{2}$ Department of Physics and Astronomy, Vanderbilt University, USA and Collaboration with Oak Ridge National \\ Laboratory, Lawrence Livermore National Laboratory and Research Institute of Atomic Reactors \\ j.h.hamilton@vanderbilt.edu
}

\begin{abstract}
The discovery of the new chemical element with atomic number $Z=117$ is presented. The isotopes ${ }^{293} 117$ and ${ }^{294} 117$ were produced in fusion reactions between ${ }^{48} \mathrm{Ca}$ and ${ }^{249} \mathrm{Bk}$. The ${ }^{249} \mathrm{Bk}$ was produced in the High Flux Isotope Reactor and chemically separated at Oak Ridge. Decay chains involving eleven new nuclei were identified by means of the Dubna Gas-Filled Recoil Separator. The measured decay properties show a strong rise of stability for superheavy nuclei toward $\mathrm{N}=184$. The reaction ${ }^{243} \mathrm{Am}+{ }^{48} \mathrm{Ca}$ was studied at three energies. Twenty one decay chains of ${ }^{288} 115$ and one decay chain of ${ }^{289} 115$ were observed to confirm the earlier discoveries of $Z=115$ and 117 .
\end{abstract}

\section{Introduction}

The shell structure of neutrons and protons strongly influences the stability of superheavy elements (SHE) see e.g., [1,2]. Without shell structure corrections, the liquid drop model predicts nuclei with $Z \geq 100$ should not exist. Theoretical predictions indicate that $\mathrm{N}=184$ should be the next spherical magic number that would give special stability to nuclei with different calculations predicting proton magic numbers of 114,120 and 126 to form an Island of Stability. The synthesis of new elements with neutron number $(\mathrm{N})$ approaching 184 provide important tests of the nuclear structure models used to predict closed spherical shells in the heaviest elements.

Cold fusion reactions (one neutron evaporation) between doubly-magic ${ }^{208} \mathrm{~Pb}$ and singly-magic ${ }^{209} \mathrm{Bi}$ target nuclei and stable neutron-rich projectiles such as ${ }^{64} \mathrm{Ni}$ and ${ }^{70} \mathrm{Zn}$ were used to synthesize new heavy elements with $Z=108-113$ and $N \leq 165$ [3, 4], stabilized by the $Z=108$ and $N=162$ shell gaps for deformed shapes. The production cross sections dropped dramatically with increasing $Z$, such that practically synthesis was not possible for heavier elements.

The Flerov Laboratory of Nuclear Reactions (JINR) $[1,5]$ pioneered a new method of synthesizing superheavy elements, with $Z \geq 112$ and neutron numbers closer to the predicted spherical shell closure at $\mathrm{N}=184$.
Four new isotopes of element $\mathrm{Z}=112$ and fourteen isotopes of new elements with $Z=113$ to 116 and 118 were identified [1] by using heavy-ion fusion reactions of doubly-magic ${ }^{48} \mathrm{Ca}$ projectiles and long lived (years) actinide targets of $\mathrm{U}$ to $\mathrm{Cm}$ and $\mathrm{Cf}$, respectively. The element with $\mathrm{Z}=117$ was missing because there is no long lived berkelium for a target. The decay properties of these 18 new nuclei provide evidence of a considerable increase in nuclear stability with increasing neutron number in the nucleus. The JINR [1,5] identification and decay properties of the $Z=112,114$ isotopes have been recently confirmed in several independent experiments [6-9]. The synthesis of ${ }^{293,294} 117(\mathrm{~N}=176,177)$ isotopes in the ${ }^{48} \mathrm{Ca}+{ }^{249} \mathrm{Bk} 4 \mathrm{n}$ and $3 \mathrm{n}$ reactions are presented here. No $(\mathrm{p}, \mathrm{xn})$ reactions to known isotopes of 116 were seen so our 117 assignment is correct. The observed longer half-lives for the $\alpha$-decay chains show increasing stability for our eleven new isotopes that end in the spontaneous fission (SF) of ${ }^{281} \mathrm{Rg}\left(\mathrm{T}_{\mathrm{SF}}=26 \mathrm{~s}\right)$ and ${ }^{270} \mathrm{Db}\left(\mathrm{T}_{\mathrm{SF}} \approx 1 \mathrm{~d}\right)$ $[10,11]$. The decisive role of the shell effects in the stability of the heaviest nuclei approaching $\mathrm{N}=184$ is strongly supported by our new data.

Studies of the ${ }^{243} \mathrm{Am}$ and ${ }^{48} \mathrm{Ca}$ reactions at three excitation energies yield 3, 6 and 12 decay chains of ${ }^{288} 115$ to confirm the discovery of this new element.

\section{Experimental procedures}

This is an Open Access article distributed under the terms of the Creative Commons Attribution-Noncommercial License 3.0, which permits unrestricted use, distribution, and reproduction in any noncommercial medium, provided the original work is properly cited. 


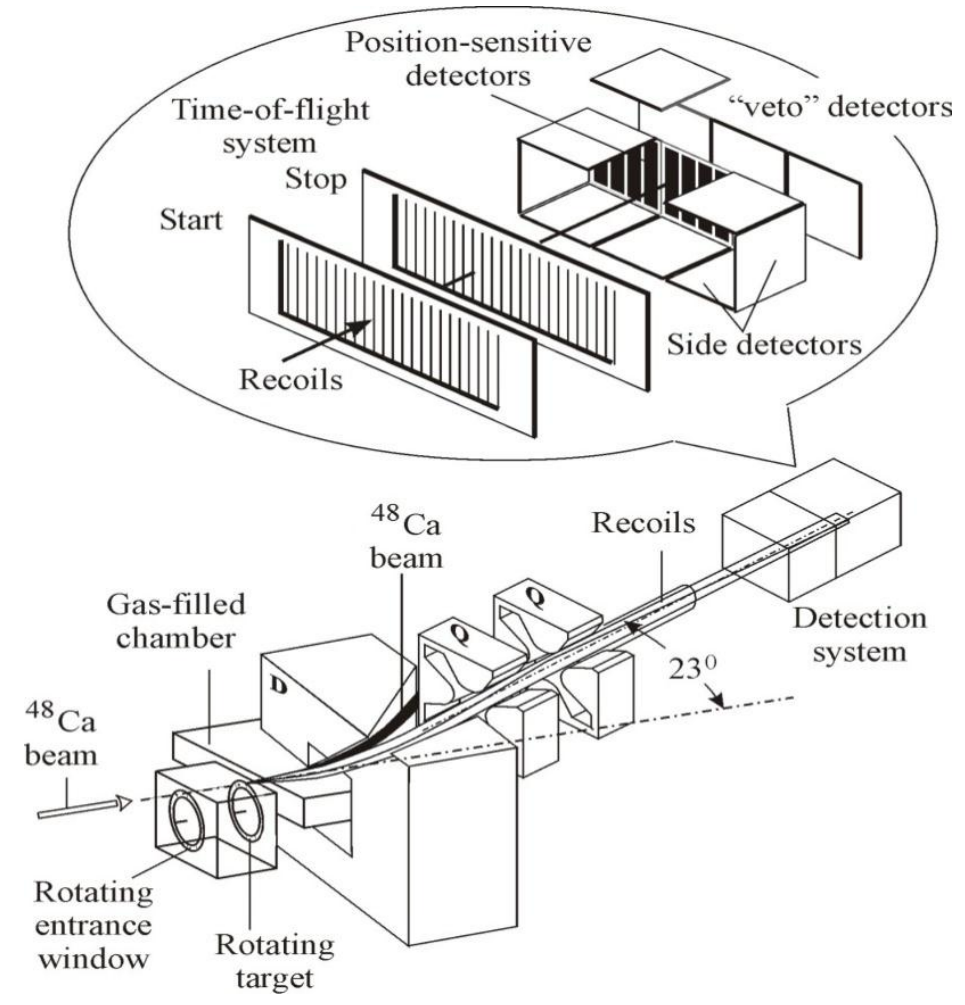

Fig. 1. Dubna Gas-Filled Recoil Separator $[1,12]$ and detector box.

The ${ }^{249} \mathrm{Bk}$ material $\left(\mathrm{T}_{1 / 2}=320 \mathrm{~d}\right)$ was produced at the High Flux Isotope Reactor (HFIR) at Oak Ridge National Laboratory (ORNL). Irradiation of $\mathrm{Cm}$ and $\mathrm{Am}$ with a flux of $2.5 \times 10^{15} \mathrm{n} / \mathrm{cm}^{2} / \mathrm{s}$ were made as part of an ongoing 8 month ${ }^{252} \mathrm{Cf}$ production campaign. After a three-month cooling period, extensive chemical separations at the Radiochemical Engineering Development Center at ORNL yielded $22.2 \mathrm{mg}$ of ${ }^{249} \mathrm{Bk}$ with only $1.7 \mathrm{ng}$ of ${ }^{252} \mathrm{Cf}$, and no other detectable impurities.

Six arc-shaped ${ }^{249} \mathrm{Bk}$ targets, each with a thickness of $0.31 \mathrm{mg} / \mathrm{cm}^{2}$, were made at the Research Institute of Atomic Reactors (Dimitrovgrad, RF) by depositing Bk nitrate onto $0.74-\mathrm{mg} / \mathrm{cm}^{2} \mathrm{Ti}$ foils. The targets mounted on the perimeter of a disk were rotated at $1700 \mathrm{rpm}$ perpendicular to the beam direction. In addition, the beam was wiggled vertically up and down over the target. The experiments were performed employing the heavy-ion cyclotron U-400 at JINR and the Dubna Gas-Filled Recoil Separator $[1,12]$.

The basic features of our work are presented here with details in refs. 10,11. After passing through the separator with an overall transmission about $35 \%$ evaporation residues (ER) were registered by a time-offlight system with a detection efficiency of $99.9 \%$. Then they were implanted in a $4 \mathrm{~cm}$ x $12 \mathrm{~cm}$ Si-detector array with 12 vertical position-sensitive strips surrounded by eight $4 \mathrm{~cm} \mathrm{x} 4 \mathrm{~cm}$ side detectors as seen in Fig. 1. The position-averaged detection efficiency was $87 \%$ of $4 \pi$ for $\alpha$-particles emitted from implanted nuclei.

The total energy of an $\alpha$-particle detected only by a side detector (its position was lost) was estimated as a sum of the energy measured by the side detector and half of the threshold energy $(\approx 0.5 \mathrm{MeV})$, with a total energy uncertainty of $\pm 0.4 \mathrm{MeV}$. The position resolution (FWHM) of the strip detector was $\leq 1.2 \mathrm{~mm}$ when registering correlated decay chains of the ER$\alpha_{1}-\alpha_{2}-\alpha_{3}-\mathrm{SF}$ type. The background rate in the detector was reduced by switching off the beam for at least 3 minutes. After a recoil signal was detected with an implantation energy expected for $\mathrm{Z}=117 \mathrm{ERs}$, followed by an $\alpha$-like signal with an energy between $10.7 \mathrm{MeV}$ and $11.4 \mathrm{MeV}$, in the same strip, within a $2.2-\mathrm{mm}$-wide position window the ${ }^{48} \mathrm{Ca}$ beam was switched off to reduce the background. A 70 day irradiation with 252$\mathrm{MeV}^{48} \mathrm{Ca}$ projectiles was performed to give a total beam dose of $2.4 \times 10^{19}$. At this energy the excitation energy of the compound nucleus ${ }^{297} 117$ is estimated to be $E^{*}=39$ $\mathrm{MeV}$, near the expected maximum for the total ER cross section (sum of $3 n$ and $4 n$ evaporation channels [1]).

\section{Results}

Five position-correlated decay chains were observed in the $252-\mathrm{MeV}^{48} \mathrm{Ca}$ irradiation. In each case, two or three $\alpha$-decays were observed between the time of arrival of the ER and the detection of SF. Fig. 2 gives the averaged decay properties of the five events assigned to the ${ }^{293} 117$ isotope. All five decay chains ended in spontaneous fission with $\mathrm{T}_{\mathrm{SF}}=26(+25,-8) \mathrm{s}$.

For the $\mathrm{E}^{*}=39 \mathrm{MeV}$ the maximum cross section is expected for the $4 n$ evaporation channel. Thus the five observed decay chains are assigned to originate from the isotope ${ }^{293} 117$. This assignment is supported by the systematics of the cross sections $\sigma_{\mathrm{xn}}\left(\mathrm{E}^{*}\right)$ measured previously for the production of superheavy isotopes with $\mathrm{Z}=108,112-116$, and 118 in ${ }^{48} \mathrm{Ca}$-induced reactions [1], 


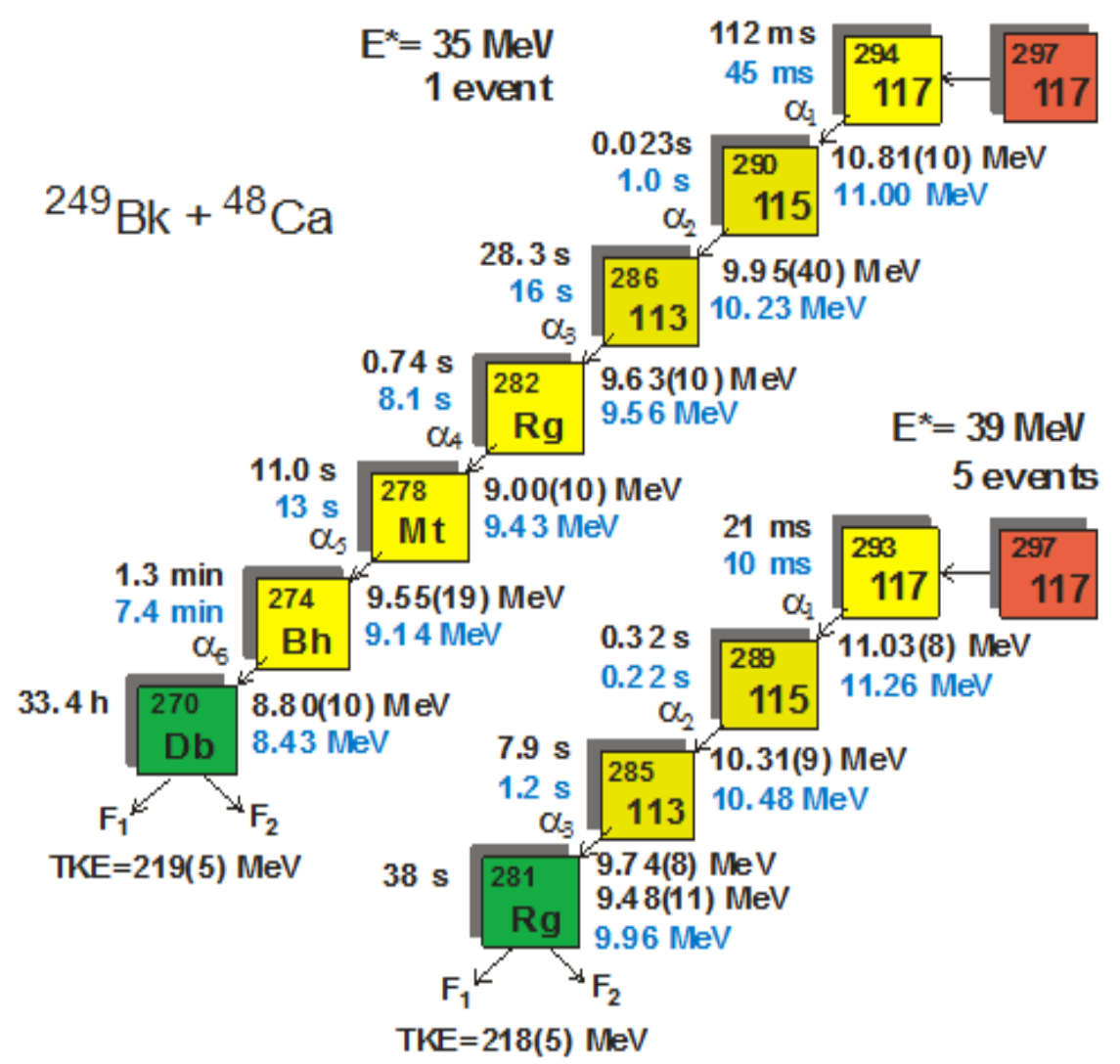

Fig. 2. Observed decay chains interpreted as originating from the isotopes $A=294$ and $A=293$ (average of five events) of the new element $Z=117$ [10]. The deduced and predicted [13] lifetimes $\left(\tau=T_{1 / 2} / \ln 2\right)$ and $\alpha$ particle energies are shown in black and blue, respectively. Taken from [10].

by calculations made for the evaporation residues of the reaction ${ }^{249} \mathrm{Bk}+{ }^{48} \mathrm{Ca}[14-16]$, and by the result of our ${ }^{249} \mathrm{Bk}+{ }^{48} \mathrm{Ca}$ experiment performed at lower beam energy (discussed next). The total numbers for random sequences [17] imitating each of the observed five decay chains were calculated to be $6 \times 10^{-6}, 10^{-3}, 10^{-5}, 3 \times 10^{-11}$ and $3 \times 10^{-11}$.

Then, the experiment was run at a ${ }^{48} \mathrm{Ca}$ energy of 247 $\mathrm{MeV}$ for 70 days with a total beam dose of $2 \times 10^{19}$. The excitation energy of approximately $35 \mathrm{MeV}$ of the compound nucleus ${ }^{297} 117$ favors the $3 n$ reaction channel. A new decay chain with six consecutive $\alpha$-decays and ending in SF was detected, see Fig 2. In this chain, the $\mathrm{Z}=111$ great-granddaughter nucleus emitted an $\alpha$-particle with $\mathrm{E}_{\alpha}=9.00 \mathrm{MeV}$ instead of undergoing $\mathrm{SF}$, followed by two more $\alpha$-transitions, and after about 33 hours, a fission event. The latter observation is significantly different from the known decay properties of ${ }^{294} 118$ and ${ }^{290-293} 116$ nuclei [1]. We assign the chain to the decay of the neighboring odd-odd nucleus ${ }^{294} 117$. The chance probability for this chain was $6 \times 10^{-11}$.

The decay properties of the neighboring ${ }^{293} 117$ and ${ }^{294} 117$ isotopes, their daughters ${ }^{289} 115$ and ${ }^{290} 115$, and granddaughters ${ }^{285} 113$ and ${ }^{286} 113$ are essentially the same but change significantly for the great-granddaughter nuclei. In spite of a strong hindrance resulting in a relatively long half-life, SF is a principal decay mode of the odd-even nucleus ${ }^{281} 111$ (see fig. 2). However, the heavier isotope ${ }^{282} 111$ undergoes $\alpha$-decay. The SF decay of ${ }^{281} 111$ can be understood by comparing the present results with the properties of the neighboring even- $Z$ nuclei. In the $\mathrm{T}_{S F}(\mathrm{~N})$ systematics, the decrease in the half-life with increasing neutron number for nuclei with $\mathrm{N}>162$ changes to a strong increase in stability as $\mathrm{N}$ approaches the spherical shell at $\mathrm{N}=184$ [18]. Minimum values of $\mathrm{T}_{S F}$ are characteristic in the transition region $\mathrm{N}=168-170$. The $\mathrm{T}_{S F}$ have minimum values because the effect of nuclear shells is at a minimum. For example, the $Z=110$ darmstadtium isotopes with $\mathrm{N}=169$ and 171 and the $\mathrm{Z}=112, \mathrm{~N}=170,172$ copernicium isotopes, undergo SF rather than $\alpha$-decay [1]. The odd-Z isotopes of elements 113 and 115 with $\mathrm{N}=169-173$ have a preference for $\alpha$-decay [19-21] because of their high hindrance of SF for nuclei with odd number of protons and the relatively low $T_{\alpha}$. Only in isotopes of elements 105 is SF observed where the $\alpha$-decay half-live exceeds $10^{5} \mathrm{~s}$ for ${ }^{268} \mathrm{Db}$. The ${ }^{249} \mathrm{Bk}+{ }^{48} \mathrm{Ca}$ reaction yields daughter nuclei that have one or two extra neutrons compared with those produced in the lower- $Z$ reactions. Approaching closer the $\mathrm{N}=184$ shell should yield a decrease in their decay energy $\mathrm{Q}_{\alpha}$ and an increase in $\mathrm{T}_{\alpha}$ with respect to the neighboring lighter isotopes at the same $\mathrm{Z}$. This behavior is clearly observed experimentally for all the isotopes with $Z \geq 111$, for the ${ }^{293} 117$ chain and in $Q_{\alpha}$ for ${ }^{294} 117$. The decay times for $Z \geq 112$ and the ${ }^{294} 117$ chain are far longer than those in the ${ }^{293} 117$ chain, see Figs. 2 and 3. By analogy with the neighboring even- $Z$ isotopes, all the nuclei in the ${ }^{293} 117$ and ${ }^{294} 117$ decay chains with $Z>111$ 


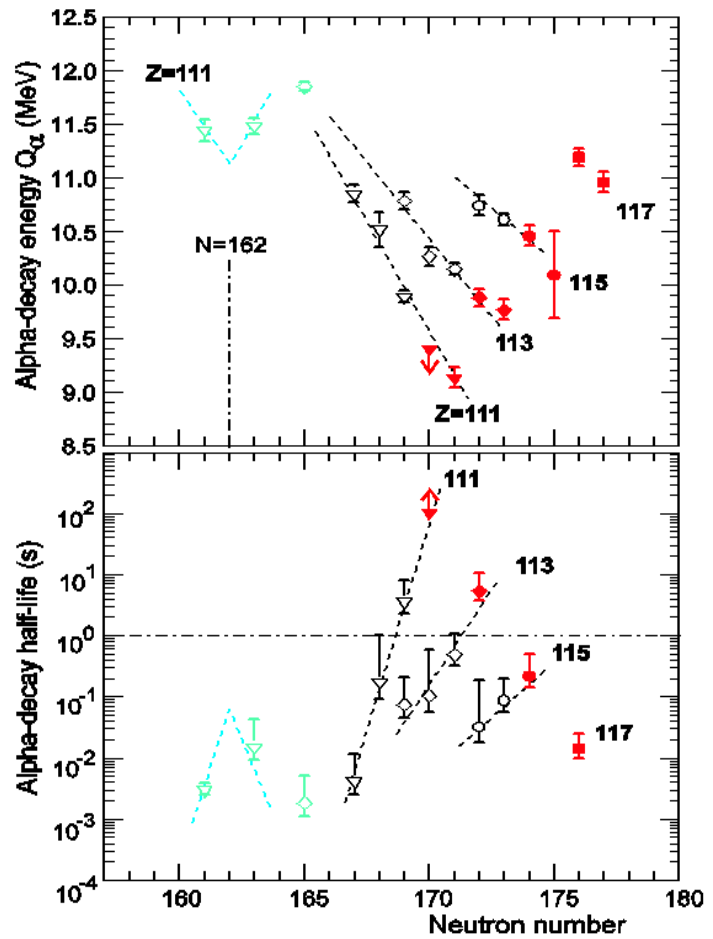

Fig. 3. a) Alpha-decay energies and b) half lives vs. neutron number for the isotopes of elements with $\mathrm{Z}=111$ 117. All the nuclides with $\mathrm{N}>165$ were produced in ${ }^{48} \mathrm{Ca}$ induced reactions. In red are shown the results obtained in the present work. In the plot (b) the values of $\mathrm{T}_{\alpha}(\exp )$ are given only for the nuclei produced in the decay of the isotope of ${ }^{293} 117$ (5 events). Figures are taken from [10].

and $\mathrm{N} \geq 172$ should undergo $\alpha$-decay. The odd proton nucleus ${ }^{281} 111(\mathrm{~N}=170)$ is in a "critical" region, and may avoid SF only because of the hindrance produced by its odd proton.

However, in spite of a hindrance of $3 \times 10^{4}$ with respect to its even-even neighbor ${ }^{282} 112$, the isotope ${ }^{281} 111$ has a probability $b_{S F} \geq 83 \%$ for SF. So, even the high hindrance caused by its odd proton does not "keep" this nucleus from SF because of the weakening of the stabilizing effect of the $\mathrm{N}=162$ and $\mathrm{N}=184$ neutron shells.

The presence of an additional unpaired neutron in its neighboring isotope ${ }^{282} 111$ further hinders SF relative to the $\alpha$-decay of this nucleus. The experimental $\mathrm{Q}_{\alpha}$ and half-lives $\mathrm{T}_{\alpha}$ are presented in Figs. 3a,b. As $\mathrm{N}$ increases, $\mathrm{Q}_{\alpha}$ decreases and $\mathrm{T}_{\alpha}$. increases. The isotopes of elements 111 and 113 exhibit an especially strong growth of $\mathrm{T}_{\alpha}$ (N). Except for ${ }^{281} 111$, all the nuclides shown in Fig. 3, are $\alpha$-emitters; with $\mathrm{T}_{\alpha}$ smaller than $\mathrm{T}_{S F}$ to indicate the high stability of the superheavy nuclei with respect to SF. The macroscopic-microscopic calculations of the masses of the superheavy nuclei [13] are in a good agreement with our experiment for all the isotopes in the decay chains of element 117 (see Fig. 2). The production cross sections for the nuclei of element 117 in the reaction ${ }^{249} \mathrm{Bk}+{ }^{48} \mathrm{Ca}$ are $\sigma=0.5(+1.1,-0.4) \mathrm{pb}$ and $\sigma=1.3(+1.5$,$0.6) \mathrm{pb}$ at $\mathrm{E}^{*}=35 \mathrm{MeV}$ and $\mathrm{E}^{*}=39 \mathrm{MeV}$, respectively. These results are similar to previously measured cross sections for the reactions of ${ }^{233,238} \mathrm{U},{ }^{237} \mathrm{~Np},{ }^{242,244} \mathrm{Pu}$,
${ }^{243} \mathrm{Am},{ }^{245,248} \mathrm{Cm}$ and ${ }^{249} \mathrm{Cf}$ targets with ${ }^{48} \mathrm{Ca}$ projectiles [1].

In order to firmly establish the discovery of the new element $\mathrm{Z}=115[19,20]$, the reaction ${ }^{243} \mathrm{Am}+{ }^{48} \mathrm{Ca} \rightarrow{ }^{288} 115$ has been studied at excitation energies $\mathrm{E}^{*}$ of 40,36 and $33 \mathrm{MeV}$ in the same way as the 117 experiment discussed above. The decay chains shown in Fig. 4 were observed at $E^{*}=40 \mathrm{MeV}$ with $\alpha$ energies and life-times the same as seen earlier [20]. At $36 \mathrm{MeV}$ six decay chains were observed and at $33 \mathrm{MeV} 12$ decay chains. The beam dosages were $3.7 \times 10^{18}, 3.3 \times 10^{18}$ and $1.7 \times 10^{19}$. All of the 21 chains have same $\alpha$ energies and half-lives within the error limits. The cross-sections for these energies are shown in Fig. 5 along with theoretical calculations [22]. Also shown is the cross section of the $2 \mathrm{n}$ channel to ${ }^{289} 115$ which was observed at $\mathrm{E}^{*}=33 \mathrm{MeV}$. This is the same isotope populated in the $\alpha$ decay of ${ }^{293} 117$. These excitation data provide clear evidence to confirm the discovery of the elements $Z=115$ and 117 .

In summary, we have synthesized a new chemical element with atomic number 117 in the fusion of ${ }^{249} \mathrm{Bk}$ and ${ }^{48} \mathrm{Ca}$. Two isotopes of element 117 , with atomic masses 293 and 294 were observed to undergo $\alpha$-decay with $\mathrm{E}_{\alpha}=11.03(8) \mathrm{MeV}$ and $10.81(10) \mathrm{MeV}$ and half lives $14(+11,-4) \mathrm{ms}$ and $78(+370,-36) \mathrm{ms}$, respectively. Their sequential $\alpha$-decay chains ended in spontaneous fission of ${ }^{281} \mathrm{Rg}\left(\mathrm{T}_{\mathrm{SF}} \sim 26 \mathrm{~s}\right)$ and ${ }^{270} \mathrm{Db}\left(\mathrm{T}_{S F} \sim 1 d\right)$, respectively. Our knowledge of the properties of odd- $Z$ nuclei in the region of the most neutron-rich isotopes of elements 105 to 117 is significantly expanded by our eleven newly identified isotopes which have increased stability with larger neutron number N. Investigations of the chemistry of superheavy elements and their place in the Periodic Table are opened up by their longer half-lives. The new isotopes, together with superheavy nuclides previously synthesized in reactions with ${ }^{48} \mathrm{Ca}$, demonstrate the critical role of nuclear shells and provide experimental verification for the existence of the predicted "Island of Stability" for superheavy elements. In a recent experiment, we confirmed the discovery of $Z=115$ by bombarding ${ }^{243} \mathrm{Am}$ with ${ }^{48} \mathrm{Ca}$ at excitation energies of 40 , 36 and $33 \mathrm{MeV}$. At these respective energies we have seen three, six, and twelve decay chains of ${ }^{288} 115$, and determined their cross sections.

We are grateful to the JINR Directorate and the U-400 cyclotron and ion source crews for their continuous support of the experiment. We acknowledged the support of the Russian Federal Agency of Atomic Energy, grants RFBR Nos. 07-02-00029, 09-02-12060, 09-03-12214, the U.S. Department of Energy through Contracts DE-AC0500OR2272 (ORNL) and DE-AC52-07NA27344 (LLNL), grants DE-FG-05-88ER40407 (Vanderbilt University) and DE-FG07-01AL67358 (UNLV). These studies were performed in the framework of the Russian Federation/U.S. Joint Coordinating Committee for Research on Fundamental Properties of Matter. 


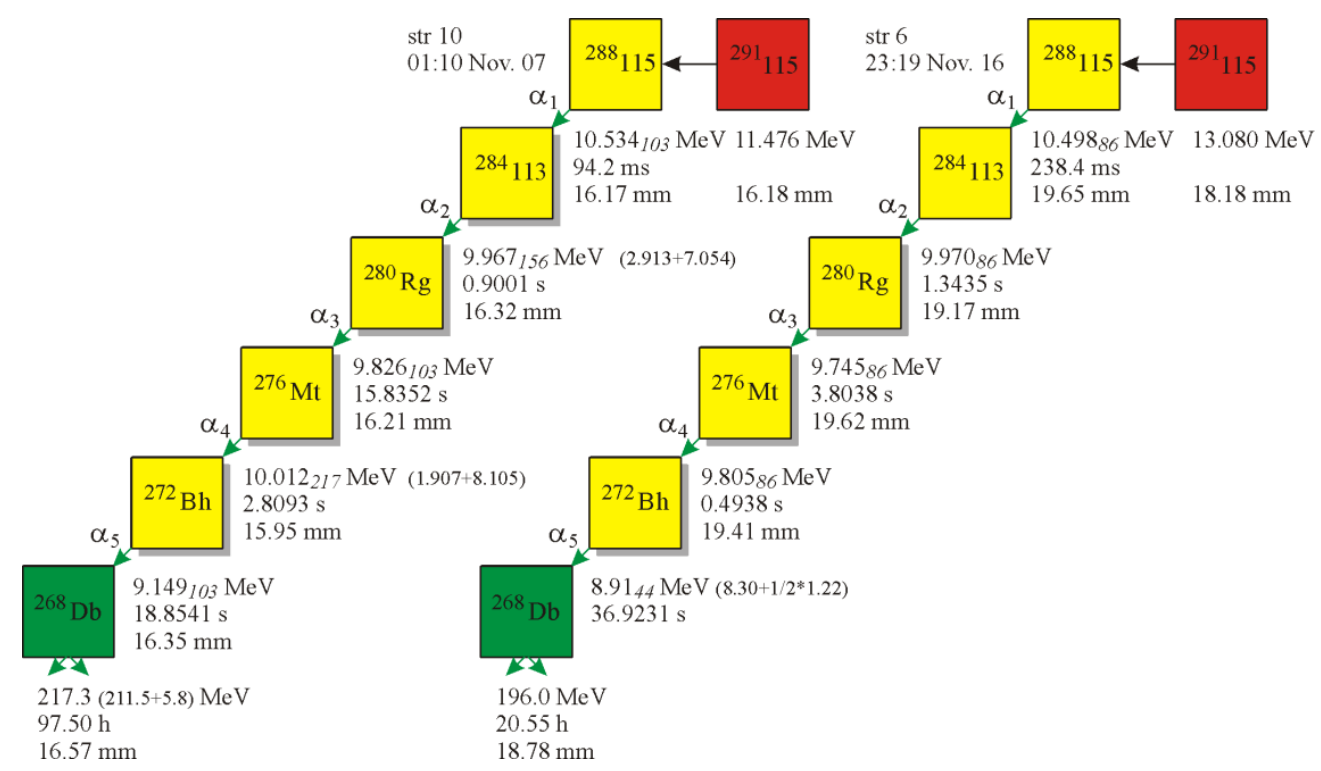

Fig. 4. Two of the three decay chains of ${ }^{288} 115$ observed in the reaction of ${ }^{243} \mathrm{Am}$ and ${ }^{48} \mathrm{Ca}$ at an excitation energy $40 \mathrm{MeV}$.

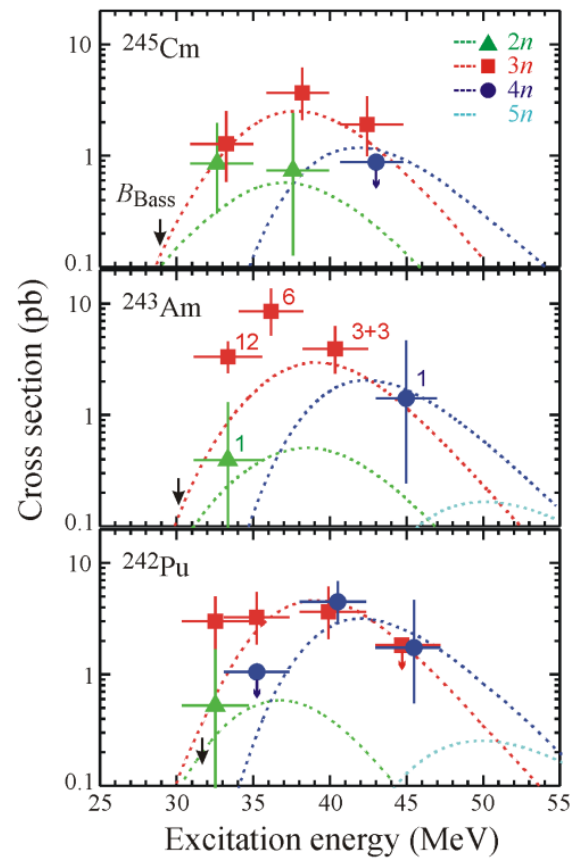

Fig. 5. Experimental and theoretical [22] (dotted curves) cross sections in $\mathrm{pb}$ for $2 \mathrm{n}, 3 \mathrm{n}, 4 \mathrm{n}$ and $5 \mathrm{n}$ reactions of ${ }^{48} \mathrm{Ca}$ with ${ }^{245} \mathrm{Cm},{ }^{243} \mathrm{Am}$ and ${ }^{242} \mathrm{Pu}$ targets. The ${ }^{243} \mathrm{Am}$ results include the earlier $[19,20]$ results and our new results.

\section{References}

1. Yu. Ts. Oganessian, Jour. Phys. G 34, R165 (2007) and earlier references therein.

2. S. Ćwiok, P.-H. Heenen and W.Nazarewicz, Nature 433, 705 (2005).

3. S. Hofmann and G. Munzenberg, Rev. Mod. Phys. 72, 733 (2000).

4. K. Morita et al., J. Phys. Soc. Jpn. 76, 045001 (2007)
5. Yu. Ts. Oganessian, this conference proceedings.

6. R. Eichler et al., Nature, 447, 72 (2007).

7. S. Hofmann et al., Eur. Phys. J. A 32, 251 (2007).

8. L. Stavsetra et al., Phys. Rev. Lett., 103, 132502 (2009).

9. Ch. E. Düllmann et al., Phys. Rev. Lett., 104, 252701 (2010).

10. Yu.Ts. Oganessian et al., Phys. Rev. Lett., 104, 142502 (2010).

11. Yu. Oganessian et al., Phys. Rev. in press (2011).

12. Yu.Ts. Oganessian et al., in Proc. of the Fourth International Conference on Dynamical Aspects of Nuclear Fission, World Scientific, Singapore, 2000, p.334.

13. A. Sobiczewski, Acta. Phys. Pol. B41, 157 (2010).

14. C. Shen et al., Int. J. Modern Phys. E 17, 66 (2008).

15. V. Zagrebaev and W. Greiner, Phys. Rev C 78, 034610 (2008).

16. Z.H. Liu and Jing-Dong Bao, Phys. Rev. C 80, 034601 (2009).

17. K. H. Schmidt et al., Z Phys. A 316, 19 (1984).

18. R. Smolanczuk, J. Skalski, and A. Sobiczewski, Phys. Rev. C 52, 1871 (1995).

19. Yu.Ts. Oganessian, Phys. Rev. C 69, 021601(R) (2004).

20. Yu. Ts. Oganessian, et al., Phys. Rev. C 72, 034611 (2005).

21. Yu.Ts. Oganessian et al., Phys. Rev. C 76 011601(R) (2007).

22. V.I. Zagrebaev, Nucl. Phys. A734 164 (2004). 